\title{
Development and optical characteristics of the macroporous silicon structures
}

\author{
Karachevtseva L.A., Lytvynenko O.A. and Stronska O.J. \\ Institute of Semiconductor Physics of NASU, 45, prospect Nauki, 03028, Kyiv, Ukraine \\ Tel.: 265 9815, FAX: 265 8243, E-mail: kartel@mail.kar.net
}

\begin{abstract}
The promising material for the $2 D$ photonic structure formation for infrared spectrum range is macroporous silicon. There was investigated the electrochemical process of the cylindrical pore formation on $n-S i$ plates for linear change of applied voltage. The experimental dependences of the regimes of macropore formation correspond to the diffusion-drift model of the nonequilibrium hole transfer for the anode thickness, that exceed the length of hole diffusion and for comparatively big macropore radii. Optical transmission of the macropore structures was measured and was equal to $10^{-2}$ comparing to that of homogeneous material; surface recombination component was estimated.
\end{abstract}

Keywords: photonic structure, macroporous silicon, electrochemical process.

Paper received 29.10.99; revised manuscript received 15.11.99; accepted for publication 04.01.00.

\section{Introduction}

Photonic band gap structures are the materials with the periodically-modulated dielectric constant. Such structures possess a frequency gap in which electromagnetic waves are forbidden analogously to the electronic band in semiconductors. Principal works with the idea of formation of the photonic band gap were published in 1987 $[1,2]$. However, this problem has been receiving dynamic development since 90s [3-9]. The spectrum of applications of the photon localization effect is wide: filters, reflectors and substrates for antennas in the microwave range [4,5]; wave guides correcting distribution of light in optical fibre [6]; zero-threshold lasers and low-loss resonators [7]; electromagnetic wave filters [3]. The effect of the photonic band gap formation opens perspectives for manufacturing photon analogs of semiconductors. The difference between photons (bosons) and electrons (fermions) determines specificity of physical phenomena in crystals with the photonic band gap and requires to revise the usual laws of absorption, emission of light, and recombination processes.

Today the most effective methods of photonic band gap formation are lithographic methods with the electrochemical procedure. Such methods permit to fab- ricate semiconductor photonic structures from nanometer to micron sizes. Especially, method of light assisted electrochemical etching permits to fabricate microporous (pore width $<2 \mathrm{~nm}$ ), mesoporous (pore with width 2-50 $\mathrm{nm}$ ) or macroporous (pore width $>50 \mathrm{~nm}$ ) structures depending upon substrate resistivity and anodization conditions. The promising material for $2 D$ photonic structure fabrication is macroporous silicon due to the contrast of the dielectric constants and periodicity.

Formation of the porous layers on silicon electrodes under anodizing in solution of hydrofluoric acid has been studying since 1950's $[10,11]$. The layers of the microporous silicon contain pores of the nanometer order size. Such structures are characterized by increased electronic band gap width [12], room temperature photoluminescence, etc. [13]. Using $n-S i$ electrodes the pores of micrometer diameter scale were obtained for the fabrication of periodical structures $[14,15]$. Mechanism of the macropore formation in silicon by the electrochemical etching in the solution of the hydrofluoric acid is connected with the generation and transfer of nonequilibrium holes to the $n-S i$ electrochemically treated surface as a result of the optical band-to-band electron-hole generation or collision ionization in strong electrical fields [14]. All holes are taking part in dissolution reaction when the 


\section{A. Karachevtseva et al.: Development and optical characteristics of the macroporous...}

hole concentration at the «semiconductor-electrolyte» interface is smaller than the concentration of the chemical reagents ( $H F$ molecules). Electrical field lines come through deepenings at the surface of the semiconductor electrode, and the pore formation takes place. But the reaction rate becomes limited by the $H F$ diffusion in electrolyte to the electrode when the hole concentration at the interface increases. So the electrode convex areas are dissolved faster, and the electropolishing process takes place. Two considered regimes are divided in $I-U$ characteristic by peak corresponded to the critical current $j_{p s}$. The process of macropores formation is self-controlled and is determined by $j_{p s}$ current density through pores. Prediction of the macroporous diameter and depth is important for the photonic structure formation. Earlier these parameters were estimated taking into account the critical current density at pore tips [14]. This model supposed that the stationary current density condition during cylindrical pore formation was equivalent to the stationary hole concentration at the pore tips. But the condition of the surface (at the tips) hole concentration cannot be satisfied taking into account the nonuniformity of the hole distribution along the sample thickness as the result of their diffusion and drift from the illuminated surface to the pore tips. So, the electrochemical process of the cylindrical pore formation on $n$-Si plates for linear change of applied voltage. Next objectives are measurements of transmittance spectra of the macroporous silicon and estimation of the surface recombination on the boundary «pore-matrix».

\section{Experimental details}

The starting material consisted of n-type silicon with resistivity $2-5 \Omega . \mathrm{cm}$. Macropores with $1-15$ micron diameters were formed due to the generation and transfer of nonequilibrium holes to the $n$-Si electrochemically treated surface as a result of the optical band-to-band electronhole generation. Initial $n-S i$ plates were chemically polished in 1:3 $\mathrm{HF}$ and $\mathrm{HNO}_{3}$ solution and anisotropically etched in $10 \%$ solution of $\mathrm{KOH}$. An ohmic contact was provided by rubbing In-Ga eutectic around the circular area exposed to the electrolyte. The samples were mounted in an electrochemical cell and connected to potentiostat by the 3-electrode scheme. The electrolyte was 5 mass percent hydrofluoric acid. The applied voltage was measured in relation to a platinum wire near the sample surface. During the electrochemical etching process the samples were illuminated by a radiation from a $100 \mathrm{~W}$ tungsten lamp.

\section{Results and discussion}

In the equilibrium state the electrochemical potential is distributed in the space charge region of semiconductor, the Helmholtz layer and a diffusive layer. According to the estimations and the experimental data the equilibrium potential is mainly determined by the semiconduc- tor space charge. So $I-U$ characteristics of silicon anode with $p$-type conductivity and strongly illuminated $n-S i$ anode are exponential and described by the Tafel equation [14]. For the semiconductor $n$-Si plates the hole current is determined by the diffusion and by the drift of the charge carriers though the $S i$ anode after intrinsic backside illumination. On the "semiconductor-electrolyte" boundary the "runoff" of nonequilibrium holes takes place. Dependencies of the nonequilibrium hole distribution through the depth of the $n-S i$ samples with various values of the hole lifetime and the electrical field strength were calculated for the macropore formation regimes in [15]. The reduction of the hole lifetime and the electrical field strength leads to the essential decrease of hole concentration along the silicon anode thickness. Regimes of the linear voltage $(U)$ dependencies on the depth of the $n$ Si samples, $U=$ const (I) and $E=$ const (II) were estimated by analogy with [15]. Voltage might be considered by linear function of sample depth $y$ between illuminated surface and pore tips: $U=a y+b$. So, hole concentration $p(y)$ in the equation for current density :

$j_{p}=e \mu_{p} p(y) E-e D_{p} \frac{d p(y)}{d y}$,

is equaled to:

$p(y)=p_{0} \exp \left[-\frac{a y+b}{2(k T / e)}\left(\sqrt{1+\frac{4(k T / e)}{\mu_{p} \tau_{p}(a+b / y)^{2}}}-1\right)\right]$

where $p_{o}$ is a hole concentration on illuminated side of $n$ Si plate, $\tau_{p}, \mu_{p}$ are hole lifetime and mobility, respectively. For regime (I) voltage being constant, and $U=b$, $a=0$, for regime (II) $E=$ const $=a, b=0$.

I. $U=$ const. It was found that stabilization of the voltage demands the maximum variation of the light intensity during the electrochemical process, and the excess hole concentration on the pore tips takes place. Such process conditions change coefficient of the electrochemical reaction and the electrical field strength in the «semiconductor-electrolyte» space charge region.

II. $\boldsymbol{E}=$ const. The illumination intensity and corresponding concentration of the nonequilibrium holes at the illuminated part of the $n$-Si plate might decrease when pore depth increase for the stationary values of $j_{p}$ and electrical field strength. Variation of the doping level of $n$-Si samples by the donor or acceptor impurities changes considerably the electron concentration, resistance, mobility and lifetime of majority and minority charge carriers. So, for the photosensitive high-ohmic $n$-Si samples $\left(\tau_{p}>5 \cdot 10^{-6} s, \rho>1 \Omega \cdot \mathrm{cm}\right)$ variation of the light intensity during the pore formation process does not exceed $10 \%$. But for the lowohmic $n$-Si samples doped by the shallow donors $\left(\tau_{p}\right.$ $\left.<5 \cdot 10^{-6} \mathrm{~s}, \rho<1 \Omega \cdot \mathrm{cm}\right)$ it is necessary to decrease the 


\section{A. Karachevtseva et al.: Development and optical characteristics of the macroporous...}

illumination intensity more than twice to save the stationary conditions of the pore formation. The experimental dependencies [14] of the macropore diameter on the electrochemical process of macropore formation were analysed for the current stabilization by the applied electrical field and the illumination intensity. The calculated data for the stabilization of the macropore formation process by the silicon anode illumination are practically independent of the doping level and are correlated with the experimental data for $\rho>1 \Omega \cdot \mathrm{cm}$. It was found that the stabilization of the hole concentration due to the light intensity change is more effective for low resistivity $n$-Si samples relatively to the electrical field variation only.

The experimental dependences of the regimes of the macropore formation correspond to the diffusion-drift model of the nonequilibrium hole transfer for the anode thickness that exceed the length of hole diffusion and for comparatively large macropore radii (Fig.1 a,b). Experimental values of the electrical field strength during the electrochemical process of the macropore formation correspond to the equality of the drift length and the sample depth between illuminated surface and pore tips.

Optical transmittance spectra of macroporous $n-S i$ were measured for $\lambda=2-25 \mu \mathrm{m}$ and were less than the sensitivity of the IR-spectrometer (IKS-31). So, absorption coefficient was more than $10^{4} \mathrm{~cm}^{-1}$. For $\lambda=1.15 \mu \mathrm{m}$ and $\lambda=3.39 \mu \mathrm{m}$ of He-Ne laser optical transmission of the macroporous structure were $10^{-2}$ apparently homoge-

a)

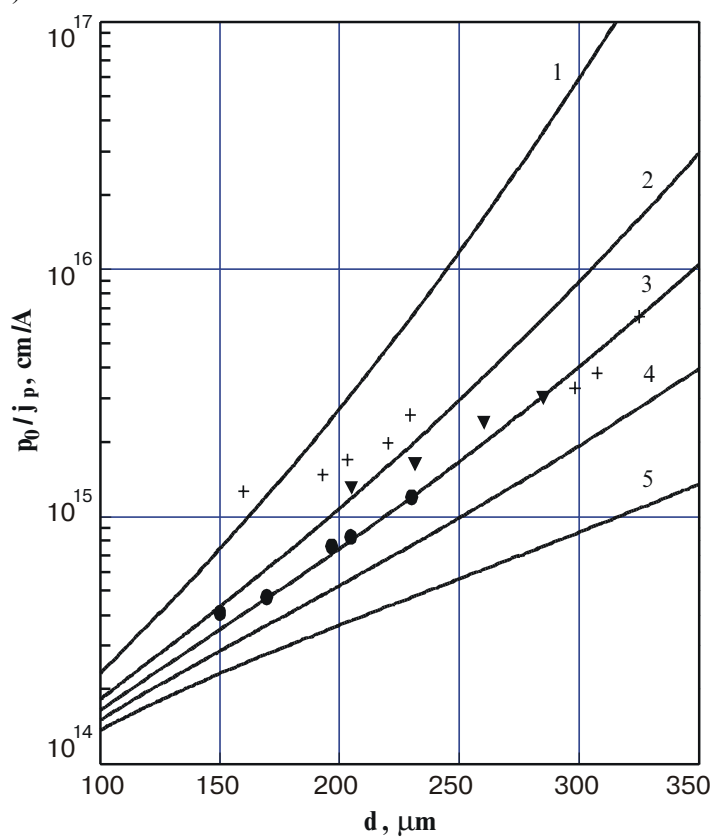

neous material (Table). Refraction coefficient for $\lambda=0.6$ $\mu \mathrm{m}$ was (2-3) $10^{-4}$ and had maximum value $10^{-1}$ in ultraviolet spectrum range. The main mechanism of the anomalous absorption is caused by diffraction of electromagnetic waves on macropores and formation of the «creeping» cylindrical waves. Really, deviations from cylindrical pore form lead to ordinary light scattering by analogy with polycrystalline, amorphous or microporous silicon, when refraction coefficient is increased.

The model was offered, and the components of the surface recombination in the two-dimensional photonic semiconductor structure were calculated using [16,17]. For the periodic structures with cylindrical inclusions (or porous) and the relation of the dielectric constants of components $\left(\varepsilon_{a} / \varepsilon_{b}\right)^{1 / 2}<1 / 3$ (that corresponds macroporous $\mathrm{Si}$ ) the surface recombination does not influence on the volumetric charge carrier lifetime with the velocity of the recombination on the boundary «inclusion - matrix» $s<10^{3} \mathrm{~cm} / \mathrm{s}$, and for the reverse relation $\left(\varepsilon_{a} / \varepsilon_{b}\right)^{1 / 2}>3$ the critical values of the surface recombination velocity are much higher and achieve the magnitude $s=10^{4} \mathrm{~cm} / \mathrm{s}$.

\section{Conclusions}

The promising material for $2 D$ photonic structure fabrication is macroporous silicon due to the contrast of the dielectric constants and periodicity. The regimes of electrochemical pore formation in macroporous silicon structures were investigated for linear change of applied

b)

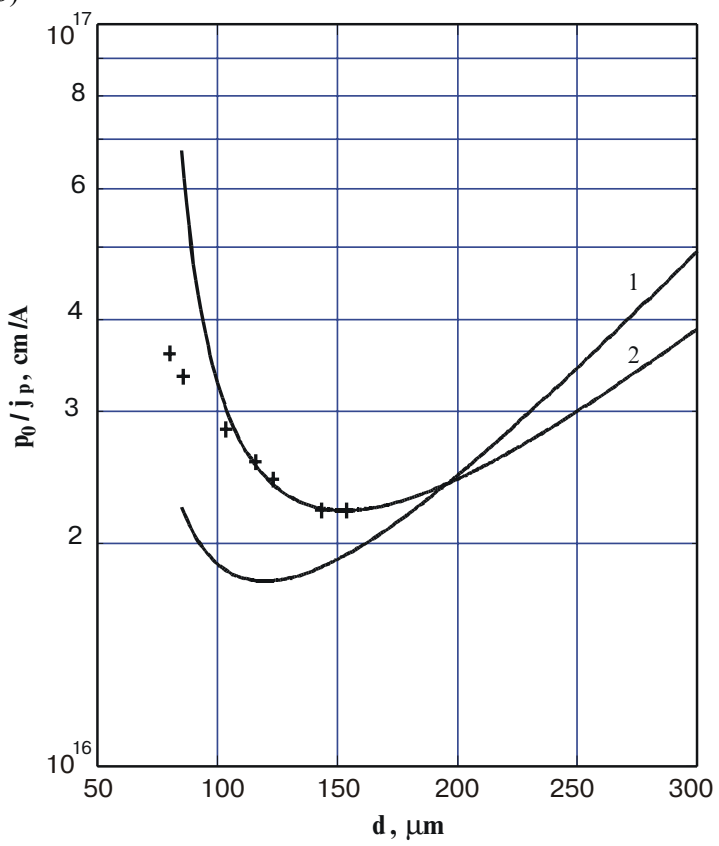

Fig. 1. Experimental (signs) and theoretical dependencies of $p_{0} / j_{p}$ regimes on silicon anode depth during pore formation for $U=$ const (1a) and $U=a d+b, \mathrm{a}=3.2 \cdot 10^{-3} \mathrm{~V} / \mathrm{cm}, b=-2.7 \cdot 10^{-2} \mathrm{~V}$ (1b) with hole lifetime:

$1 \mathrm{a}-\tau_{p}, 10^{-7}$ s: $1-3,2-5,3-7,4-10,5-20$;

$1 \mathrm{~b}-\tau_{p}, 10^{-7} \mathrm{~s}: 1-7,2-10$. 
L. A. Karachevtseva et al.: Development and optical characteristics of the macroporous...

Table. Optical transmission of macroporous n-Si structures

\begin{tabular}{|c|c|c|c|c|c|c|c|c|}
\hline \multirow[t]{2}{*}{$\begin{array}{l}\text { № of } \\
\text { samples }\end{array}$} & \multicolumn{3}{|c|}{$\begin{array}{l}\text { Regimes of } \\
\text { electrochemical } \\
\text { etching }\end{array}$} & \multicolumn{3}{|c|}{ Pore parameters } & \multicolumn{2}{|c|}{ Transmission, $\%$} \\
\hline & $\mathrm{U}, \mathrm{V}$ & $\mathrm{I}, \mathrm{mA}$ & $\mathrm{t}, \min$ & Depth, $\mu \mathrm{m}$ & $\begin{array}{l}\text { Diame- } \\
\text { ter, } \mu \mathrm{m}\end{array}$ & $\begin{array}{l}\text { Concen- } \\
\text { tration, } \\
\mathrm{cm}^{-2}\end{array}$ & $\lambda=1.15 \mu \mathrm{m}$ & $\lambda=3.39 \mu \mathrm{m}$ \\
\hline 1 & \multicolumn{6}{|c|}{ substrate (monocrystal n-Si) } & 2.1 & 3.2 \\
\hline $\begin{array}{l}2 \\
3\end{array}$ & $\begin{array}{l}0.6 \\
1.0\end{array}$ & $\begin{array}{c}15 \\
5\end{array}$ & $\begin{array}{l}40 \\
50\end{array}$ & $\begin{array}{l}25 \\
25\end{array}$ & $\begin{array}{c}5-10 \\
2-7\end{array}$ & $\begin{array}{l}1.5 \cdot 10^{4} \\
1.9 \cdot 10^{5}\end{array}$ & $\begin{array}{l}0.05 \\
0.03\end{array}$ & $\begin{array}{l}0.05 \\
0.04\end{array}$ \\
\hline $\begin{array}{l}4 \\
5\end{array}$ & $\begin{array}{l}1.0 \\
0.6\end{array}$ & $\begin{array}{c}5 \\
15\end{array}$ & $\begin{array}{c}60 \\
120\end{array}$ & $\begin{array}{l}40 \\
60\end{array}$ & $\begin{array}{c}7-12 \\
10-15\end{array}$ & $\begin{array}{l}8.7 \cdot 10^{5} \\
6.7 \cdot 10^{4}\end{array}$ & $\begin{array}{c}0.02 \\
0.008\end{array}$ & $\begin{array}{l}0.03 \\
0.02\end{array}$ \\
\hline
\end{tabular}

voltage. The relationship between parameters of the electrochemical process was analysed within the diffusion-drift theory. The constant field strength regime is favourable for stable macropore formation. The experimental dependences of the regimes of macropore formation correspond to the diffusion-drift model of the nonequilibrium hole transfer for anode thicknesses that exceed the length of hole diffusion and for comparatively big macropore radii. Optical transmittance spectra of macroporous $n-S i$ were measured for $\lambda=2-25 \mu \mathrm{m}$. Optical transmission of the macropore structure were $10^{-2}$ apparently homogeneous material. For the periodic structures with cylindrical inclusions (or porous) and the relation of the dielectric constants of components $\left(\varepsilon_{d} / \varepsilon_{b}\right)^{1 / 2}<$ $1 / 3$ (that corresponds to macroporous $\mathrm{Si}$ ) the surface recombination does not influence on the volumetric charge carrier lifetime with the velocity of the recombination on the boundary «inclusion - matrix» $s<10^{3} \mathrm{~cm} / \mathrm{s}$.

\section{References}

1. E. Yablonovitch, Inhibited spontaneous emission in solid state physics and electronics // Phys. Rev. Lett. 58(20), pp. 2059$2062(1987) /$

2. S. John, Strong localization of photons in certain disordered dielectric superlattices // Phys. Rev. Lett. 58(23), pp. 24862489 (1987).

3. L. A. Karachevtseva, A. V. Lyubchenko, Materials with Photonic Band Gap // Optoelectronics and Semiconductor Technics. 32, pp. 150-161 (1997).

4. E. R. Brown, C. D. Parker, E. Yablonovitch, Radiation Properties of a Planar Antenna on a Photonic-crystal Substrate // J. Opt. Soc. Am. B. 10(2), pp. 404-407 (1993).

5. E. R. Brown, C. D. Parker, O. V. McMahon, Effect of surface composition on the radiation pattern from a photonic-crystal planar-dipole antenna // Appl. Phys. Lett. 64(24), pp. 33453347 (1994).
6. T. A Birks, D. M. Atkin, G. Wylangowski, P. Russel, P. Roberts, 2D Photonic Band Gap Structure in Fibre Form, article in Photonic Band Gap Materials, Eds. C. M. Soukoulis, pp. 437-444, Kluwer Academic Publishers (1996).

7. P. R. Villeneuve, M. Piche, Photonic band gaps in two-dimensional square and hexagonal lattices // Phys. Rev. B. 46(8), pp. 4969-4972 (1992).

8. S. John, J. Wang, Quantum optics of localized light in a photonic band gap // Phys. Rev. B. 43(16), pp.12772-12789 (1991).

9. K. Busch and C. M. Soukoulis, Energy Transport Velocity in Random Media, article in Photonic Band Gap Materials, Eds. C. M. Soukoulis, pp. 667-678, Kluwer Academic Publishers (1996).

10. D. R. Turner, Electropolish Silicon in Hydrofluoric Acid Solutions // J. Electrochem. Soc. 105(7), pp. 402-408 (1958).

11. M. I. I. Theunissen, Etch Channel Formation during Anodic Dissolution of N-Type Silicon in Aqueos Hydrofluoric Acid // J. Electrochem.Soc. 119(3), pp. 351-359 (1972).

12. L. T. Canham, Silicon quantum wire array fabrication by electrochemical and chemical dissolution of wafers // Appl. Phys.Lett. 57(10), pp. 1046-1048 (1990).

13. J. M. Martinez-Duart, V. P. Parkhutik, R. Guerrero-Lemus, and J. D. Moreno, Electroluminescent Porous Silicon // Advanced Materials 7(2), pp. 226-228 (1995).

14. V. Lehmann, H. Foll, Formation Mechanism and Properties of Electrochemically Etched Trenches in n-Type Silicon // J. Electrochem. Soc. 137(2), pp. 653-659 (1990).

15. L. A. Karachevtseva, O. A. Litvinenko, E. A. Malovichko, Stabilization of Electrochemical Formation of Macropores in $n$-Si // J. Theor.and Experim.Chem. 34(5), pp. 314-318 (1998).

16. N. N. Grigoriev, L. A. Karachevtseva, K. R. Kurbanov, Growth Dislocation Influency on Electron Lifetime in nHgCdTe // Fizika and Tekhnika Polupr. 25(3), pp. 464-466 (1991).

17. L. A. Karachevtseva, A. V. Lyubchenko, Peculiarities of Surface Recombination in Semiconductor Structures with Photonic Band Gap // Ukrainian Physical Journal. 43(10), pp. 1254-1258 (1998). 